\title{
TRES TEOREMAS FUNDAMENTALES DE LA TEORÍA DEL DISEÑO DE ESTRUCTURAS
}

\author{
(THREE FUNDAMENTAL THEOREMS OF STRUCTURAL DESIGN THEORY)
}

Jaime Cervera Bravo, Dr. Arquitecto

Fecha de recepción: $12-1 X-88$

\section{RESUMEN}

En este articulo, tras recordar dos conocidos teoremas de Maxwell, 1890, y Michell, 1904, se presenta un importante teorema de la teoria de diseño de estructuras, tanto por su generalidad teórica, como por sus implicaciones prácticas; la estructura de diseño óptimo para un problema dado es también la más rígida de entre todas las correspondientes a diseños alternativos, entendiéndose que las alternativas lo son de forma, manteniendo el mismo grado de aprovechamiento del material (idénticas tensiones de trabajo). De este modo, concebir modificaciones (de forma) en un diseño que reduzcan la deformabilidad de la estructura significa simultáneamente disminuir la inversión en material en ésta. Se adopta una formulación explícita para tal teorema, apuntado como propiedad de las estructuras óptimas por Parkes, 1965.

\section{SUMMARY}

This article, after mentioning the well-known theories of Maxwell (1890) and Michell (1904), presents an important theorem on structural design, both for its theoretical generality as well as for its practical implications. The best structural design from among many alternatives is the most rigid one, the alternatives being understood to be alternatives in shape, maintaining the same use of materials (identical working tensions). In this fashion, the conception of modifications (in shape) in a design which reduces deformation of the structure means simultaneous reduction of materials. An explicit formulation is adopted for this theorem, considered as a property of optimal structures by Parkes (1965).

\section{INTRODUCCIÓN}

Es suficientemente conocido el diferente contenido del diseño y del análisis, y esta diferencia se aplica entre otros campos al de la definición de estructuras: poder analizar una estructura para asegurar que cumple los requerimientos estructurales exige un elevado grado de diseño previo de la misma: En general el análisis explora objetos definidos al efecto de validarlos o invalidarlos, y dichos objetos deben estar descritos con tanta mayor precisión, previamente al análisis, cuanto mayor es la precisión del método adoptado.

La tarea de definir estructuras exige, por lo tanto, un proceso iterativo de diseño-análisis en el que, por otra parte, las decisiones en las primeras etapas de la definición son precisamente las más importantes.

(C) Consejo Superior de Investigaciones Científicas

Licencia Creative Commons 3.0 España (by-nc)
Con herramientas de análisis adecuadas, y mediante un sistema de prueba y error, puede llegar a encontrarse una estructura suficientemente estable, segura, rígida, y compatible con otros requerimientos de uso, e incluso que se sitúe en un coste razonable, siempre que se destinen a ello los medios y el tiempo necesarios. Los cuatro primeros requerimientos son obviamente ineludibles, pero para evaluar el último debe considerarse también el propio coste del proceso de diseño. En función de los medios a emplear puede llegarse a un punto en que el ahorro de materiales sea menor que el aumento de coste del proceso de diseño, punto que depende del número de objetos iguales que hayan de producirse.

La experiencia del proyectista puede acortar el camino de prueba y error permitiéndole producir rápidamenhttp://informesdelaconstruccion.revistas.csic.es 
te diseños válidos y razonables en cuanto a coste, pero por el contrario limita la búsqueda de soluciones alternativas.

La teoría del diseño estructural trata de racionalizar el proceso de diseño estableciendo, mediante el análisis de soluciones genéricas, reglas que permitan llegar en el menor tiempo y al menor coste posible a soluciones razonables. Cuando hablamos de teoria del diseño estructural nos referimos al diseño general de la estructura; al final del proceso será precisa una etapa de diseño específico en la que se produzca la documentación necesaria para la ejecución de la estructura.

En el pasado, el énfasis de la investigación ha sido puesto en la depuración de los métodos de análisis, énfasis mediante el que se han logrado niveles de precisión muy elevados. Sin embargo, los primeros intentos realizados en la línea de formalizar una teoría del diseño (Maxwell, 1890, Michell, 1904,...) no han sido valorados ni continuados adecuadamente. El grupo de profesores formado en torno a la docencia de Cálculo de Estructuras III y Proyectos de Estructuras de la Escuela Técnica Superior de Arquitectura de Madrid, de Ia Universidad Politécnica, ha realizado diversos avances en ese terreno (1). Este artículo enuncia tres teoremas básicos para dicha teoría. Finalmente se exponen algunas reflexiones sobre la misma.

\section{TRES TEOREMAS FUNDAMENTALES DE LA TEORÍA DEL DISEÑO DE ESTRUCTURAS}

\subsection{Definiciones previas}

En primer lugar definiremos como Problema de Max. well el de definir una estructura capaz de poner en equilibrio entre si a las fuerzas de un sistema tal que tanto la posición como la magnitud de las mismas están definidas. En dicho problema es evidente que la resultante y el momento resultante de dicho sistema de fuerzas es nulo, pues de otro modo el equilibrio no es posible. La función de la estructura será conectar dichas fuerzas entre sí, constituir la ligazón física que permita materializar tal equilibrio.

Debe quedar claro que se trata de fuerzas definidas también en posición, y que dicho sistema incluye, tal como está formulado el problema, todas las fuerzas ex. ternas del mismo, tanto acciones como reacciones.

Dicho problema es, asimismo, un problema teórico en la medida en que la existencia y la forma de la estructura tienen una influencia considerable en la magnitud y posición de tales fuerzas (y ello sin considerar que muchas de ellas son imprevisibles, por más que exis. tan formas convencionales normadas de considerarlas). Sin embargo es posible, en tipologías dadas de estructuras, acotar las fuerzas a que se somete la estructu- ra, por lo que puede en éstas plantearse uno o varios Problemas de Maxwell que caractericen su comportamiento.

Llamaremos Estructura de Maxwell a la estructura que resuelve un Problema de Maxwell y que cumple la condición siguiente:

- está constituida por elementos que trabajan uniaxilmente, en tracción o compresión.

Llamamos finalmente Estructura estricta a toda estructura de Maxwell tal que:

- en todo punto de la estructura el dimensionado es estricto, ie. si la estructura está realizada con un material dado, en todas las secciones de la misma el material se halla sometido a la máxima tensión compatible con la seguridad ( $\sigma$ de servicio en todos los puntos).

Se trata nuevamente de estructuras teóricas, en la medida en que en la realidad no es usual cumplir dichos requerimientos: Los diseños no son estrictos, tanto por razones derivadas de la necesidad de rigidez (deformaciones limitadas, estabilidad,...), como por razones constructivas (dimensionados por sección constante,...).

Las piezas en flexión no encajan con facilidad en la descripción, mantienen tensiones variables en la sección, muchos de cuyos puntos se encuentran sometidos a estados planos no uniaxiles,... Existen, sin embargo, estructuras en flexión que sí pueden hacerlo, y se puede intentar modelizar la pieza mediante conceptos asociados a alguna de dichas estructuras, con las salvedades que sean necesarias (por ejemplo emulando los estados biaxiles de cortadura por una doble familia de estados de tracción-compresión,....).

Existen estructuras y componentes estructurales que basan su comportamiento en estados bi o triaxiles. Sin embargo forman una familia que supone un porcentaje limitado del total de estructuras construidas, y en ellas a menudo el uso de materiales heterogéneos lleva a trabajar en éllas con estados uniaxiles, pese al global de la estructura: piénsese en los armados en placas o láminas de hormigón, que suponen un importante componente estructural sometido a tracción (o compresión en algunos casos) uniaxil.

En estas condiciones podemos definir un importante concepto, asociado en gran medida al coste de la estructura:

Cantidad de estructura, W (2) es la integral extendida a toda la estructura de los productos $|\mathrm{N}| \cdot \mathbf{d s}$, siendo 


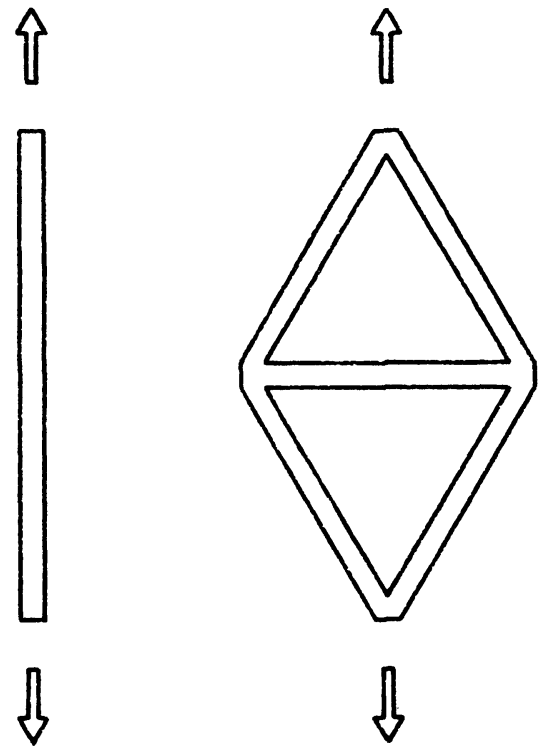

Fig. 1.-Para ambos esquemas estructurales (1 $T$ a $1 \mathrm{~m}$ de distancia) las magnitudes $W$ y $M$ valen

$$
\begin{array}{ll}
W=1 \mathrm{~m} . t & W=1.666 \mathrm{~m} . \mathrm{t} \\
M=1 \mathrm{~m} . \mathrm{t} & M=1.00 \mathrm{~m} \cdot \mathrm{t}
\end{array}
$$

N el esfuerzo en la "sección" y ds el elemento longitudinal de pieza en el punto considerado.

$$
w=\int|N| \cdot d s
$$

Se consideran valores absolutos de modo que, ya se trate de tracción o compresión, se obtengan valores positivos. Tal cantidad acumula todos los productos de esfuerzo por longitud correspondientes a todas las "barras" de la estructura, y tiene dimensiones físicas de trabajo. (Fig. 1).

La cantidad de estructura es una medida del volumen de la estructura no asociada al material utilizado: en efecto, sea un material de comportamiento simétrico, en tracción y compresión, cuya tensión de trabajo sea $\sigma$. Para una estructura estricta, en toda sección el área será:

$$
A=|N| / \sigma
$$

y el elemento de volumen

$$
d V=A \cdot d s
$$

de modo que el volumen de la estructura será:

$$
\mathbf{V}=\int \mathbf{A} \cdot \mathbf{d s}=\int|\mathbf{N}| / \sigma \cdot \mathbf{d s}=\mathbf{W} / \sigma
$$

si admitimos $\sigma$ constante.
De este modo en una Estructura Estricta el volumen de la estructura es directamente la "cantidad de estructura" dividida por la Tensión de Servicio. Si no es posible asegurar idéntica tensión en todos los puntos, el volumen será mayor que dicho cociente, pero estará gobernado por la magnitud $\mathbf{W}$.

Veremos más adelante que en el caso de una estructura formada, bien por materiales diferentes en compresión o tracción, bien por un material de comportamiento no simétrico en ambos estados y siendo $\sigma_{\mathrm{t}} \# \sigma_{\mathrm{c}}$ las tensiones en servicio en tracción y compresión, el Volumen de la Estructura $\mathbf{V}$ es una función lineal de $\mathbf{W}$.

\section{Teorema de Maxwell}

Para toda Estructura de Maxwell, que resuelve un mis. mo problema de Maxwell, la cantidad

$$
\mathbf{M}=\int \mathbf{N} \cdot \mathbf{d s}
$$

es función de las fuerzas aplicadas y de sus puntos de aplicación, e independiente de la forma de la estructura, siendo la misma para todas éllas.

Nótese que aquí no se trata de valores absolutos de $\mathbf{N}$, sumando por lo tanto compresiones y tracciones con signos opuestos. Nótese, asimismo, que la afirmación se hace para toda estructura de Maxwell - no necesariamente de dimensionado estricto- que resuelve idéntico problema.

Para demostrar dicho Teorema basta aplicar el Teorema de los Trabajos Virtuales a una estructura tal:

Por dicho teorema, si sometemos a una estructura en equilibrio a un movimiento o deformación arbitrario, el trabajo total realizado por fuerzas externas e internas es nulo.

Supongamos pues una deformación consistente en expandir uniformemente la estructura en torno al origen de coordenadas, que permanece fijo, ampliando las dimensiones lineales I en un factor $(1+e)$. Denotaremos por $\mathbf{e}_{i}$ al vector desplazamiento de todo punto $i$ en tales circunstancias. Tales vectores forman una radiación de centro en el origen y de magnitud proporcional a la distancia de cada punto al mismo.

En esta situación el trabajo realizado por las fuerzas exteriores será $\Sigma F_{i} \cdot \mathbf{e}_{i}$, suma de los productos escalares fuerza desplazamiento. Si desglosamos en componentes:

$$
\Sigma F_{i} \cdot e_{i}=\Sigma F_{x i} \cdot X_{i} \cdot e+\Sigma F y_{i} \cdot Y_{i} \cdot e+\Sigma F_{z i} \cdot Z_{i} \cdot e
$$


Por otro lado la deformación interior en cada componente de la estructura será e, constante en toda ella.

El trabajo de deformación interno a la estructura será: $\mathbf{U}=\int \sigma \cdot \mathbf{e} \cdot \mathbf{d V}=\mathbf{e} \cdot \int \sigma \cdot d V=\mathbf{e} \cdot \int \mathbf{N} \cdot(\mathrm{dVIA})=$ $=e \cdot \int \mathbf{N} \cdot d s$

y por lo tanto si el trabajo total debe ser nulo resulta

$M=\int N \cdot d s=\Sigma o F_{x i} \cdot X_{i}+\Sigma F y_{i} \cdot Y_{i}+\Sigma F_{z i} \cdot Z_{i}$

Nótese que $\sigma$ puede ser variable sin que ello afecte al razonamiento.

En las anteriores expresiones, suponiendo una estructura dada a la que se aplica la expansión uniforme e, es evidente observar que $U$, y por lo tanto $\Sigma F_{i} \cdot e_{i}$ no dependen del sistema de ejes de referencia elegido. Co. mo a su vez $\Sigma F_{i} \cdot e_{i}$ no depende de la estructura ele. gida, resulta ser $\mathbf{M}$ independiente de ésta, es decir es una constante del Problema, que no depende de su solución.

De dicho teorema se derivan im.portantes corolarios:

1) En un problema de Maxwell, la diferencia entre la cantidad de estructura utilizada en tracción y compresión permanece constante para todas las estructuras que lo resuelven, por lo que reducir la parte de estructura que trabaja en compresión conlleva simultáneamente reducir la parte en tracción, y vice. versa.

En efecto, desglosando la cantidad de estructura $W$ en dos partes, una $\mathbf{W}^{+}$en tracción y otra $\mathbf{W}^{-}$en compresión resulta:

$$
\begin{aligned}
& \mathbf{W}=\mathbf{W}^{+}+\mathbf{W}^{-} ; \\
& \mathbf{M}=\mathbf{W}^{+}-\mathbf{W}^{-} \text {(constante) }
\end{aligned}
$$

Reducir uno de los términos en la segunda expresión exige reducir el primero, al efecto de que se mantenga la diferencia. Por ello:

2) Si se minimiza una de las dos partes de compresión o de tracción de una estructura se minimiza la estructura.

3) Una estructura de Maxwell, sólo traccionada o sólo extendida, es ya una estructura mínima, y todas las diferentes estructuras que puedan proponerse en estas condiciones para el problema de Maxwell dado son equivalentes.
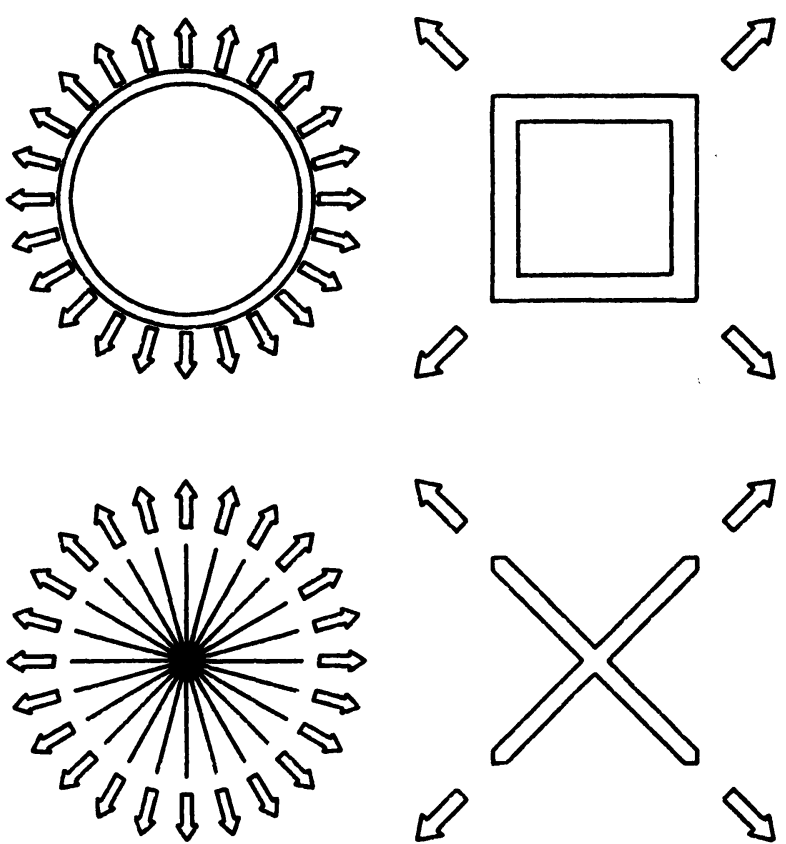

\$
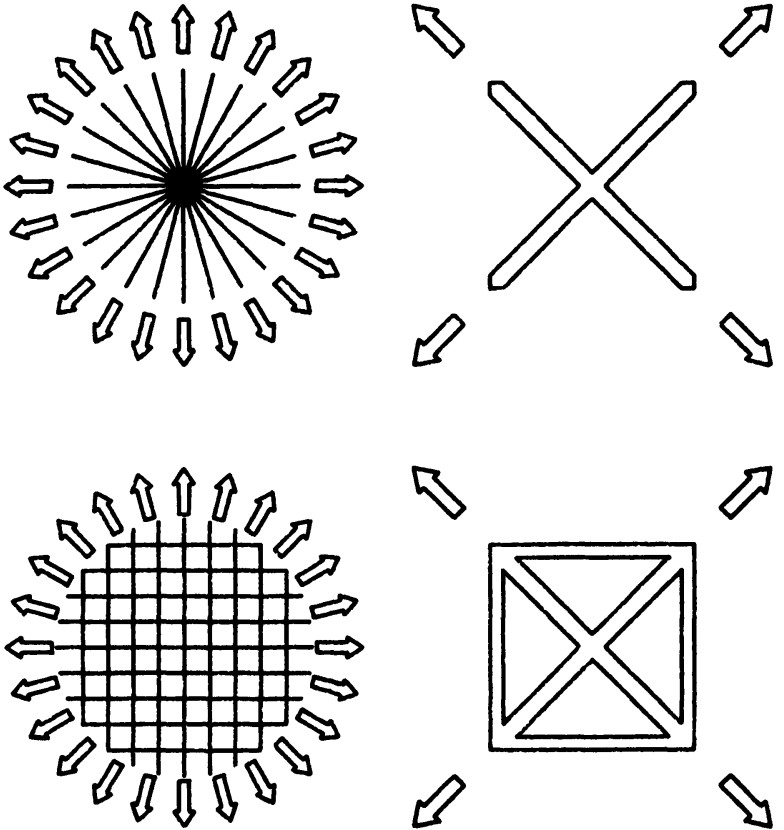

§
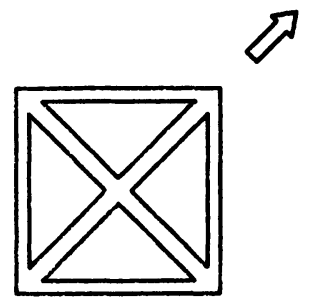

C
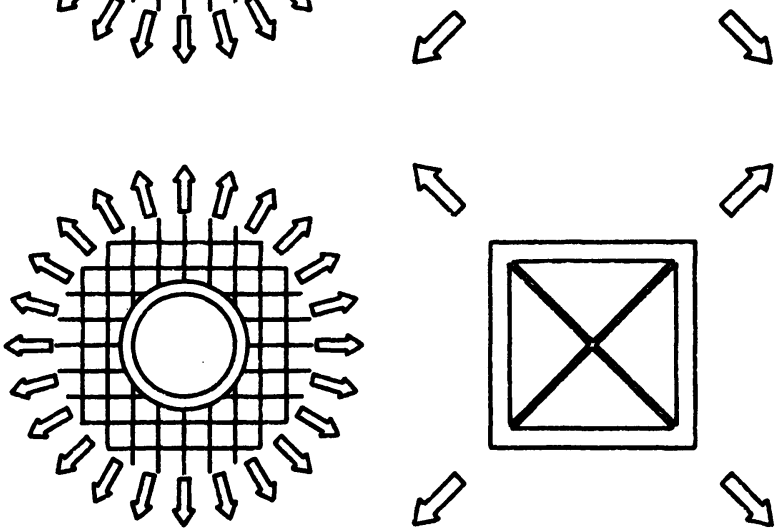

C

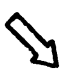

Fig. 2.-Las cuatro soluciones estructurales (para cada problema) son idénticas en consumo de material y deformación.

Es fácil ver asimismo que en estas condiciones, y si admitimos pequeños desplazamientos en las fuerzas para acomodarse a la deformación, todas las estructuras estrictas posibles tienen igual deformación, y por lo tanto no existen siquiera problemas de compatibilidad en soluciones hiperestáticas, en la medida en que sometiendo a todas las secciones a idéntica $\sigma$ se asegura que estén sometidas a idéntica deformación $\epsilon$, y por lo tanto todo el esquema estructural experimenta una expansión (contracción) uniforme (y por lo tanto compatible) igual para todos los esquemas (Fig. 2). 
Podemos ver ahora que en el caso de una estructura dimensionada estrictamente y formada, bien por materiales diferentes en compresión o traccción, bien por un material de comportamiento no simétrico en ambos estados y siendo $\sigma_{\mathrm{t}}$ y $\sigma_{\mathrm{c}}$ las tensiones máximas admisibles en tracción y compresión en valores absolutos, el Volumen de la Estructura $\mathbf{V}$ es una sencilla función lineal de $W$, de modo que la estructura de mínimo volumen es también la de menor cantidad de estructura.

En efecto el volumen será:

$v=\int|N| / \sigma \cdot d s=\int d W / \sigma$

(expresión que no es más que una generalización de [2] y por lo tanto

$V=1 / \sigma_{\mathrm{t}} \int \mathrm{d} \mathrm{W}^{+}+1 / \sigma_{\mathrm{c}} \int \mathrm{d} \mathrm{W}^{-}=\mathrm{W}^{+} / \sigma_{\mathrm{t}}+\mathrm{W}^{-} / \sigma_{\mathrm{c}}$

como

$\mathbf{M}=\mathbf{w}^{+}-\mathbf{w}^{-}$, resulta

$\left(\sigma^{t}-\sigma^{c}\right) \mathbf{M}=\left(\sigma^{t}-\sigma^{c}\right) \mathbf{W}^{+}-\left(\sigma^{t}-\sigma^{c}\right) \mathbf{W}^{-}$

y como

$2 \cdot \sigma_{t} \cdot \sigma_{c} \cdot V=2 \cdot \sigma_{c} \cdot W^{+}+2 \cdot \sigma_{t} \cdot W^{-}$

resulta, sumando ambas expresiones,

$2 \cdot \sigma_{t} \cdot \sigma_{c} \cdot \mathbf{V}+\left(\sigma^{t}-\sigma^{c}\right) \mathbf{M}=\left(\sigma_{t}+\sigma_{c}\right)\left(\mathbf{W}^{+}+\mathbf{W}^{-}\right)=$

$$
=\left(\sigma_{t}+\sigma_{c}\right) W
$$

de este modo

$\mathbf{V}=\frac{\left(\sigma_{t}+\sigma_{c}\right)}{2 \cdot \sigma_{t} \cdot \sigma_{c}} W-\frac{\left(\sigma_{t}-\sigma_{c}\right)}{2 \cdot \sigma_{t} \cdot \sigma_{c}} M$

Es fácil ver que en problemas de sólo tracción o sólo compresión $\mathbf{W}$ y $\mathbf{M}$ coinciden, salvo signos, mientras que en los problemas de sólo flexión M es nula (Fig. 3).
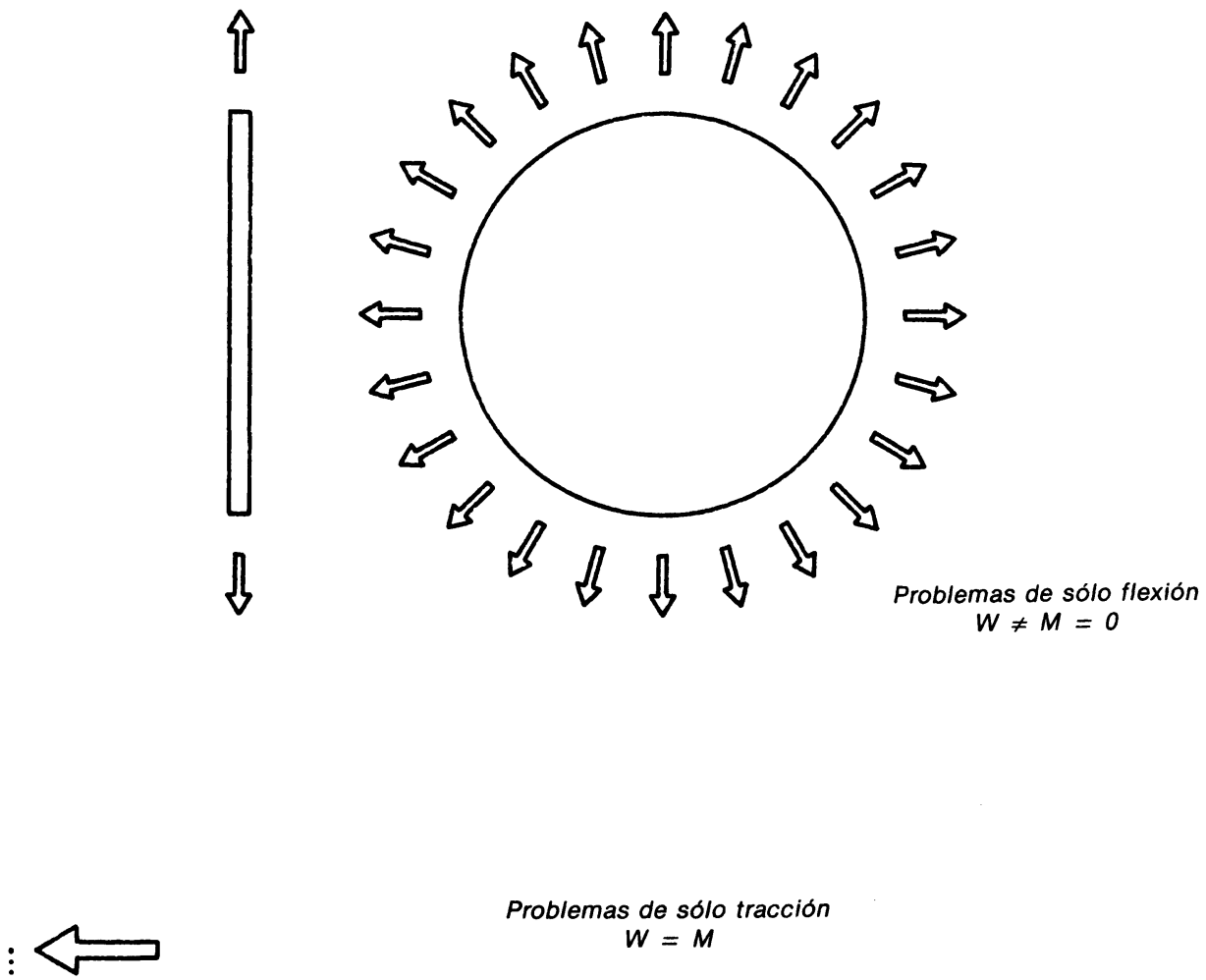

Problemas de sólo tracción $W=M$ 


\section{Teorema de Michell}

(Veamos a continuación una formulación del mismo).

Una estrutura estricta alcanza el límite de economia (es estructura mínima) si el espacio en el que está situada puede ser sometido a una deformación (virtual), tal que los alargamientos 0 acortamientos unitarios se incrementan igualmente en todas las piezas (con el mismo valor y en el signo original), y en valor no menor que en el cambio unitario de longitud de cualquier elemento del espacio considerado.

Si el espacio considerado se extiende al infinito, en todas las direcciones, el volumen de tal estructura es minimo con relación a todos los posibles diseños, y en caso contrario minimo en relación a los diseños que pueden considerarse incluidos en el mismo contorno finito.

Considérese el espacio delimitado por un contorno dado encerrando todas las estructuras estrictas interiores a ese contorno que puedan concebirse para un cierto problema de Maxwell. Considérese dicho espacio sometido a una deformación (virtual) arbitraria, y tal que el valor absoluto de las deformaciones principales (virtuales) en todo punto del espacio sean menores que un cierto valor e dado. (En toda dirección del espacio el valor absoluto del alargamiento o acortamiento unitario $\mu$ es: $|\mu| \leq$ e). Por el principio de los trabajos virtuales, la variación en la energía de deformación de cada estructura es igual al trabajo virtual de las fuerzas exteriores $\delta \mathbf{U}$ y por lo tanto igual para todas ellas. Podemos escribir, pues,

$$
\delta \mathbf{U}=\int \mu \cdot \sigma \cdot \mathbf{d} \mathbf{V}
$$

igual para todas las estructuras consideradas.

$$
\begin{gathered}
\delta \mathbf{U}=\int \mu \cdot \sigma \cdot \mathbf{A} \cdot \mathbf{d s}=\int_{\cdot} \mu \cdot \mathbf{N} \cdot \mathbf{d s} \leq \int|\mu| \cdot|\mathrm{N}| \cdot \\
\cdot \mathbf{d s} \leq \int \mathrm{e} \cdot|\mathrm{N}| \cdot \mathbf{d s}
\end{gathered}
$$

de modo que para todas ellas,

$$
\delta U \leq e \int d W
$$

De este modo los valores posibles para la cantidad de estructura W, incluyendo el correspondiente a la estructura mínima, quedan acotados inferiormente por $\delta \mathbf{U} / \mathbf{l}$.

Ahora bien, si es posible encontrar una estructura cuyas deformaciones se incrementen en la deformación virtual, i: con el mismo valor $(|\mu|=$ e) en todos los puntos, y ii: en el mismo sentido que las deformaciones originales, en el desarrollo anterior los signos $\leq$ se modifican $\mathrm{a}=$, por lo que dicha estructura tiene como cantidad de estructura la citada cota inferior, y es por lo tanto la estructura mínima (Fig. 4).

De la clase de estructuras que pueden ser sometidas con éxito a una deformación test del tipo citado, Michell presenta dos subclases:

1) las estructuras sometidas a esfuerzos de igual signo en todas sus barras (para éstas la deformación test es una contracción o una dilatación uniforme del espacio);
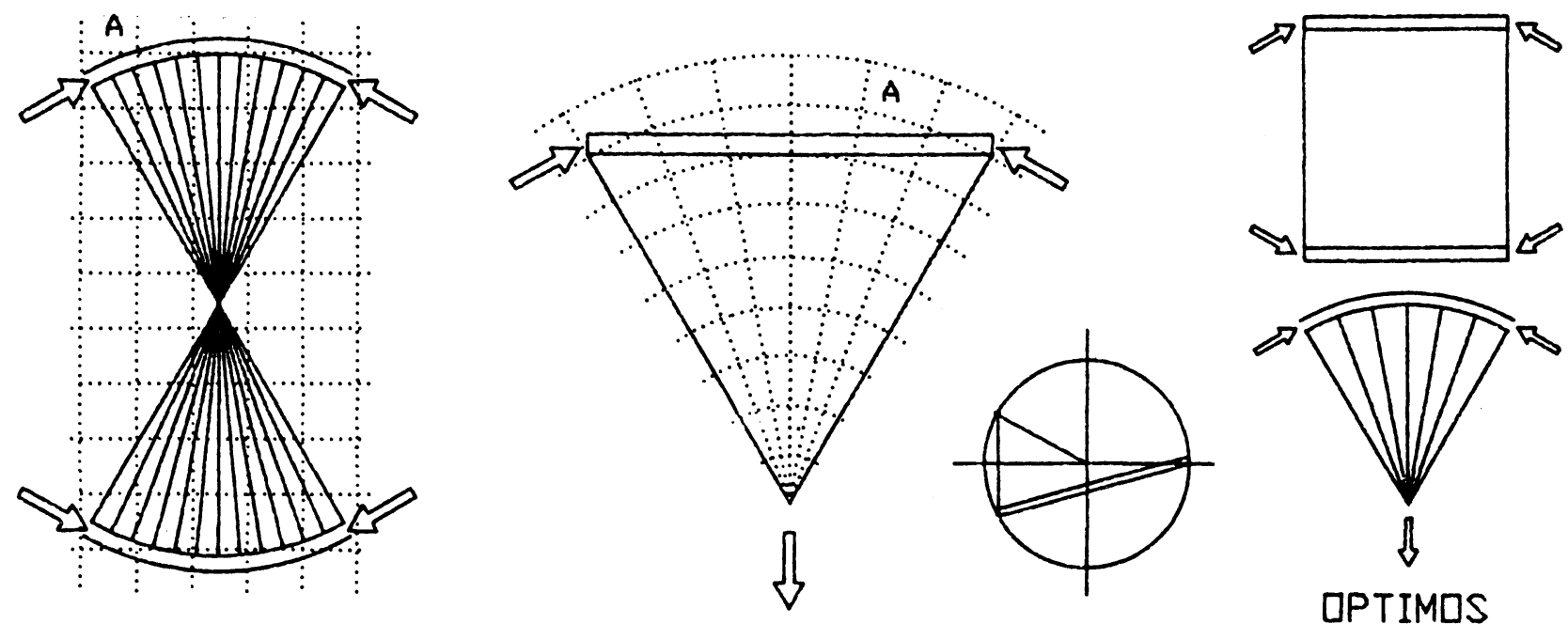

Fig. 4.-En la deformación test (círculo de Mohr adjunto) la dirección de una barra en un punto como el A no "aprovecha" toda la posible deformación. 

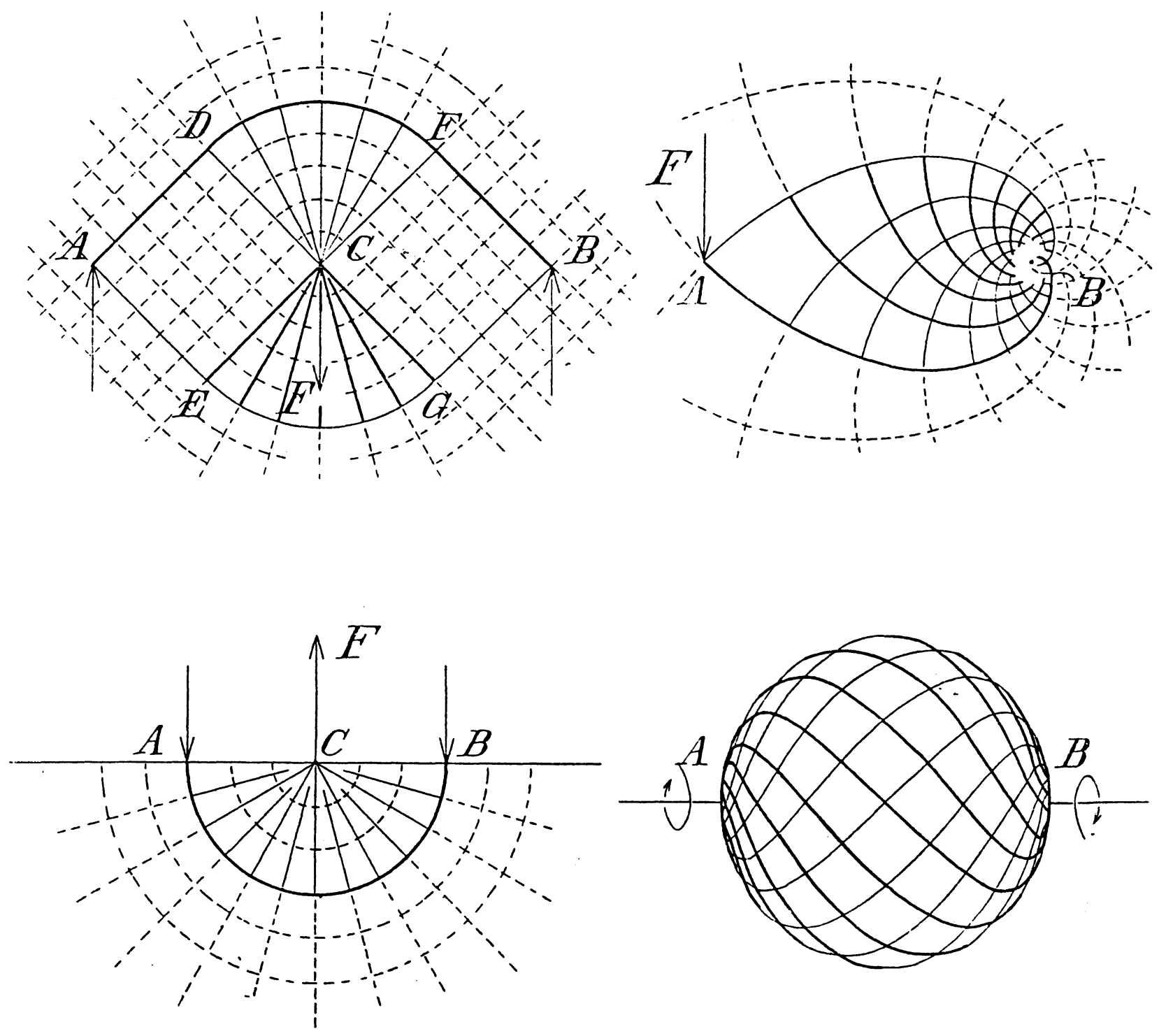

Fig. 5.-Estructuras de Michell.

2) las estructuras formadas por barras en direcciones ortogonales antes y después de la deformación, para las que la deformación test es igual en valor, y de igual u opuesto signo en tales direcciones (Fig. 5).

Veamos, por último, el tercer teorema.

\section{Teorema de la mínima deformación}

De entre todas las estructuras estrictas que resuelven el mismo problema de Maxwell, todas aquellas que tienen igual cantidad de estructura tienen igual deforma. ción; la deformación aumenta con la cantidad de estructura del esquema elegido, y, finalmente, la estructura mínima (la de menor cantidad de estructura) es a su vez la más rígida.
Para poder demostrar el Teorema hemos de definir previamente cómo describimos de una forma abstracta y uniforme la deformación para esquemas estructurales que pueden ser muy diferentes. Se trata de usar una norma coherente del desplazamiento, un escalar que permita la comparación y que sea acorde a la "deformabilidad" que se asigna más o menos intuitivamente a cada estructura.

Es usual, en esquemas sencillos (pórtico de una altura, viga de un vano,...) usar como medida de la deformación de la estructura un desplazamiento de ésta, y en particular el desplazamiento en la posición y dirección de la carga principal ("bajo" la carga principal) (Fig. 6), y cuando no es ese el caso, un valor que es proporcional a éste para tipos comparables de estructuras (Figura 7). 

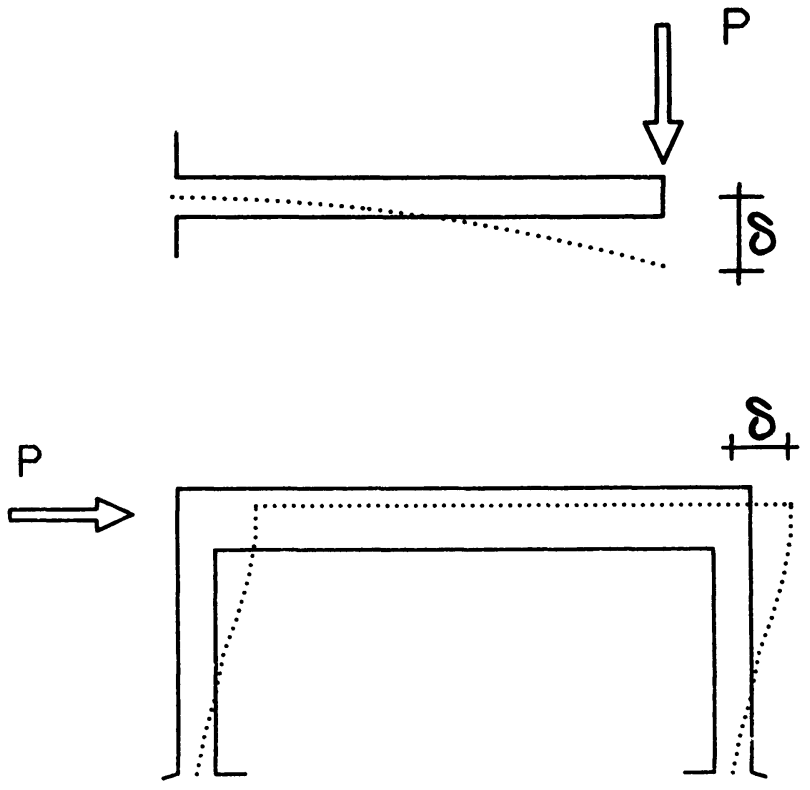
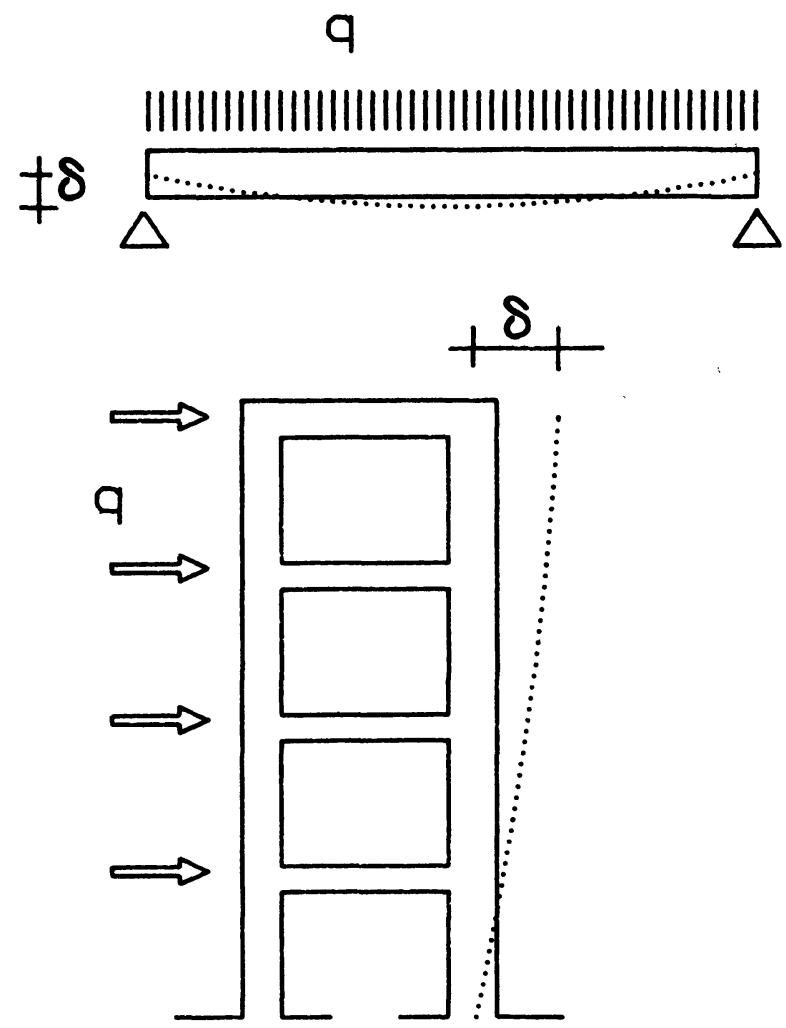

Figs. 6 y 7.-Medidas usuales de la deformación. La flexibilidad de la estructura suele medirse como $\delta / P$, $\delta / q$.

Para problemas de Maxwell, los únicos parámetros fijos son la posición y magnitud de las cargas, por lo que es apropiado usar los desplazamientos "bajo" éstas, debiendo ser combinados en forma apropiada todos éllos en un único escalar. Una forma de hacerlo es sumarlos escalarmente asignando pesos a cada uno, bien iguales, bien que denoten una contribución diferente a la suma según su importancia respectiva (pesos diferentes según la importancia). Se trataría, pues, de una medida del tipo:

$$
\delta_{\theta}=\Sigma \alpha_{i} \cdot \delta_{1}
$$

Siendo $\delta_{\theta}$ la medida de la deformación de la estructu$\mathrm{ra}, \alpha_{\mathrm{i}}$ el peso asignado a cada desplazamiento y $\delta_{\mathrm{i}} \mathrm{ca}$ da uno de éllos. La forma más evidente de asociar un peso a cada desplazamiento es hacerlo mediante la magnitud de la carga aplicada en el punto correspondiente. Se reproduce asi la idea intuitiva de que las cargas mayores son las de mayor importancia en la descripción del comportamiento global de la estructura. De este modo

$$
\delta_{\theta}=\Sigma \mathbf{P}_{\mathbf{i}} \cdot \delta_{\mathrm{i}}
$$

Ahora bien, es fácil ver que tal magnitud es la pérdida de energía potencial de las fuerzas que constituyen el

(c) Consejo Superior de Investigaciones Científicas Licencia Creative Commons 3.0 España (by-nc) problema debido a la deformación de la estructura. Corresponde, pues, a un concepto claro, adecuado en los casos sencillos citados con anterioridad, y utilizable de forma totalmente general.

Por tanto, adoptando como deformación de la estructura el valor de la pérdida de energía potencial de las fuerzas, que sobre élla actúan, basta medir este valor y compararlo en estructuras diferentes. Dicho valor, considerado desde el punto de vista de la deformación de la estructura, no es más que la integral extendida a todo su volumen del producto Tensión. Deformación (suma de la energía de deformación y de la energía complementaria) (Fig. 8).

$\delta_{\theta}=\mathbf{U}_{\mathbf{p}}=\int \sigma \cdot \epsilon \cdot \mathbf{d V}=\int \sigma \cdot \epsilon \cdot \frac{\mathrm{dW}}{\sigma}=\int \epsilon \cdot d \mathbf{W}$

Tratándose de comparar diseños estrictos de idéntico material, como los valores de tensión y deformación son constantes e iguales para todos los diseños considerados, y eso en todas y cada una de sus secciones, resulta que la deformación de la estructura es proporcional en estructuras estrictas a la cantidad de estructura de éstas.

http://informesdelaconstruccion.revistas.csic.es 


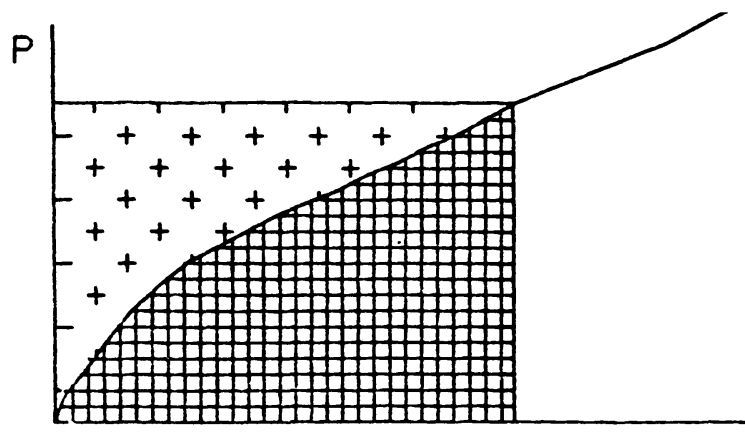

$+$

"Energia complementaria" "Energía de deformación"

Fig. 8.-

De ello se deduce que estructuras de igual cantidad de estructura son igualmente deformables, y que la estructura minima (de menor cantidad de estructura) es asimismo la más rígida.

Ha de repetirse que se trata de la comparación entre estructuras de forma diferente propuestas para idéntico problema de Maxwell, y que usan el mismo material con idéntico aprovechamiento local (iguales tensiones y deformaciones locales): es evidente que para cualquiera de tales estructuras podria aumentarse la rigidez (a la vez que el consumo de material) disminuyendo la tensión de trabajo (y el aprovechamiento del material).

Si se usan materiales o tensiones diferentes en tracción y compresión, el proceso es algo más complicado, pero se llega a idéntico resultado:

$\mathbf{U}_{\mathrm{p}}=\int q \cdot \epsilon_{\mathrm{t}} \cdot \mathbf{d} \mathbf{V}+\int \sigma_{\mathrm{c}} \cdot \epsilon_{\mathrm{c}} \cdot \mathbf{d} \mathbf{V}=\epsilon_{\mathrm{t}} \cdot \mathbf{W}^{+}+\epsilon_{\mathrm{c}} \cdot \mathbf{W}$;

$2 \cdot U_{p}=2 \cdot \epsilon_{t} \cdot W^{+}+2 \cdot \epsilon_{c} \cdot W^{-} ;$

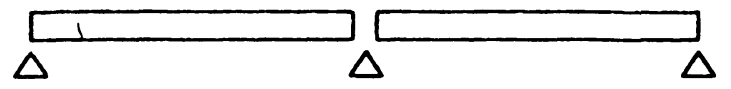

$>$
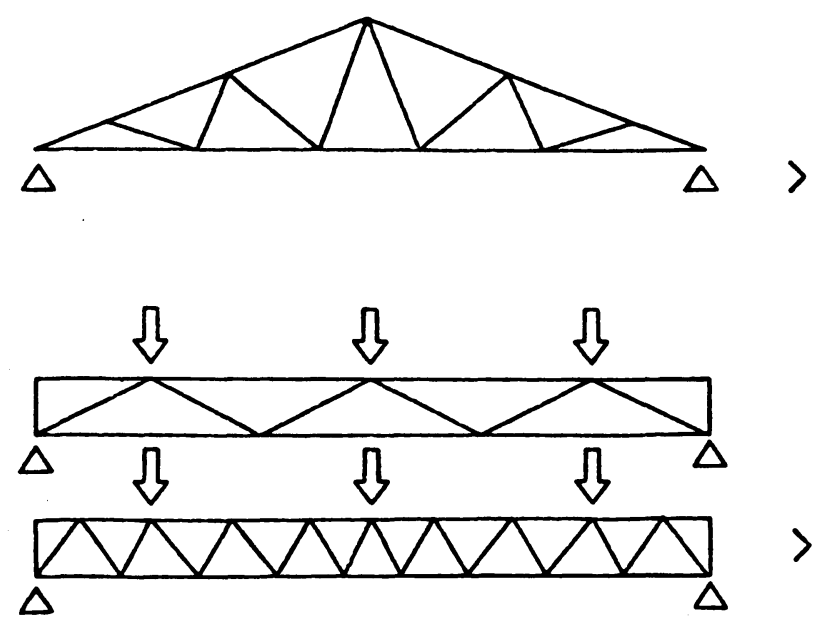

$$
\begin{aligned}
& \left(\epsilon_{1}-\epsilon_{\mathrm{c}}\right) \cdot \mathbf{M}=\left(\epsilon_{\mathrm{t}}-\epsilon_{\mathrm{c}}\right) \cdot \mathbf{W}^{+}-\left(\epsilon_{\mathrm{t}}-\epsilon_{\mathrm{c}}\right) \cdot \mathbf{W}^{-} ; \\
& \begin{array}{r}
\mathbf{2} \cdot \mathbf{U}_{\mathrm{p}}-\left(\epsilon_{\mathrm{t}}-\epsilon_{\mathrm{c}}\right) \cdot \mathbf{M}=\left(\epsilon_{\mathrm{t}}+\epsilon_{\mathrm{c}}\right) \cdot\left(\mathbf{W}^{+}+\mathbf{W}^{-}\right)= \\
\quad=\left(\epsilon_{\mathrm{t}}+\epsilon_{\mathrm{c}}\right) \cdot \mathbf{W}
\end{array} \\
& \mathbf{U}_{\mathrm{p}}=\frac{\left(\epsilon_{\mathrm{t}}+\epsilon_{\mathrm{c}}\right)}{\mathbf{2}} \cdot \mathbf{W}+\frac{\left(\epsilon_{\mathrm{t}}-\epsilon_{\mathrm{c}}\right)}{\mathbf{2}} \cdot \mathbf{M}
\end{aligned}
$$

Tal teorema permite justificar completamente la ligazón existente entre menor coste y menor deformación en los casos conocidos: vigas continuas frente a vigas apoyadas, cerchas trianguladas 0 arcos de canto óptimo, etcétera (Fig. 9).

Puede verse que las magnitudes $\mathbf{W}$ y $\mathbf{M}$ revisten una importancia crucial en la descripción del diseño de una estructura, gobernando tanto su volumen como su deformación.

Cabe añadir, además, que $\mathbf{W}$ (cantidad de estructura) es mejor medida de la inversión en estructura requerida por un diseño que $\mathbf{V}$ (volumen de material), en la medida en que no depende del material elegido para materializar dicho diseño, siendo sus magnitudes exclusivamente fuerzas y recorridos correspondientes al esquema estructural adoptado.

Del teorema anterior pueden deducirse importantes corolarios:

1) Para minimizar la estructura de un problema de Maxwell bastará obtener el esquema estructural que minimice la deformación de la misma (siempre que se utilice el material en su máximo aprovechamiento).
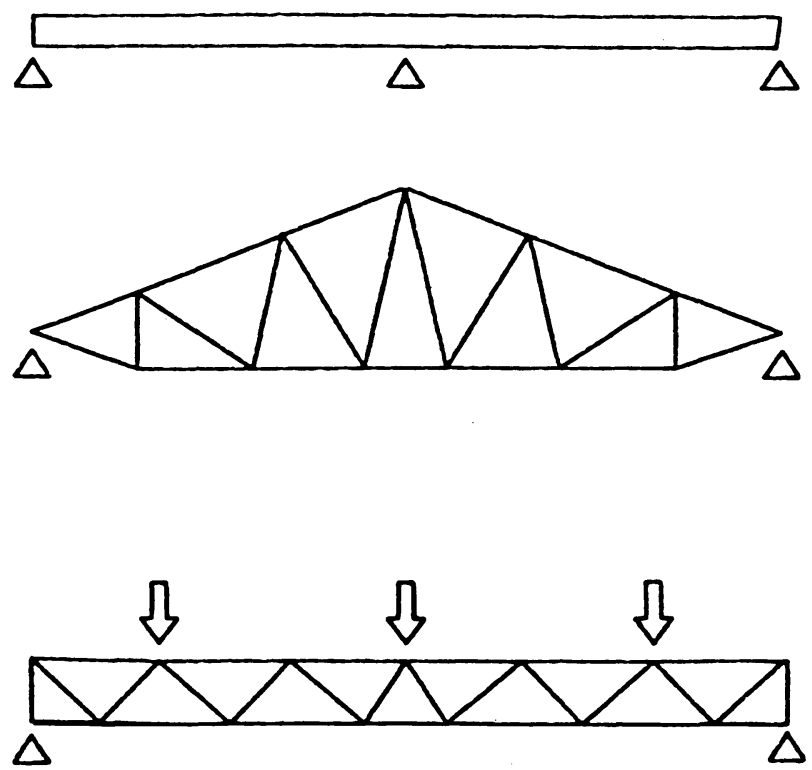

Fig. 9.-Todas las soluciones de la columna izquierda son mayores (en coste) y más deformables que las correspondientes soluciones de la columna derecha para idéntico aprovechamiento del material (igual tensión de trabajo). 
2) Disminuir la rigidez de un esquema estructural conlleva aumentar su cantidad de estructura, y, en la medida en que el coste depende de aquélla, aumentar el coste final de la estructura.

3) Si para un tipo estructural dado, el límite en que tal tipo deja de ser utilizable por razones económicas (en competencia con otros tipos) corresponde a un esquema en el que se cumplen los requerimientos de deformación sin necesidad de disminuir las tensiones en sus secciones, dichos requerimientos de deformación se cumplen igualmente en todos los esquemas utilizables de dicho tipo, pues corresponderán a estructuras de menor coste y, por tanto, de menor cantidad de estructura y, por ende, de menor deformación.

\section{Unas reflexiones finales}

En primer lugar desearía que los teoremas anteriores permitan llamar la atención sobre las posibilidades que ofrece una teoría del diseño de estructuras.

En segundo lugar quiero señalar que en la medida en que la realidad es geométrica, y en la medida en que las herramientas que se utilizan en las etapas iniciales del diseño son herramientas geométricas (dibujos, croquis,...), introducir la reflexión sobre el diseño estructural en esas etapas exige (y es posible hacerlo) establecer conceptos y herramientas de reflexión de tipo geométrico: de otro modo no podrian ser utilizadas con facilidad en el análisis y contraste de las diferentes opciones (no sólo estructurales) en juego. De este modo la teoría deberá tener un carácter marcadamente geométrico, describiendo cuestiones gobernadas en parte por las formas, por las proporciones, y en parte por los tamaños. El análisis, aplicado a tipologías genéricas de estructuras, permite establecer dichas herramientas.

\section{NOTAS:}

(1) Ha de citarse la labor pionera desarrollada por los profesores Ricardo Aroca y José Luis de Miguel en este terreno.

(2) Término acuñado por Ricardo Aroca. Con anterioridad ha sido utilizado el de "trabajo estructural".

\section{BIBLIOGRAFIA}

1. J. C. Maxwell, 1890: Scientific papers, Cambridge Univ. Press. London Vol. 2.

2. Michell A. G. M., 1904: "The limits of Economy of Material in Frame-structures" en Philosophical Magazine. S.6, Vol. 8, N. 47 Nov. 1904.

3. Parkes E. V. V., 1965: "Braced Frameworks". Pergamon Press, Oxford.

4. José Luis de Miguel, 1974. Tesis Doctoral: "Trabajo estructural: Un nuevo escalar en el diseño de estructuras". ETSAM. Universidad Politécnica de Madrid.

5. Hemp W. S., 1973: "Optimum Structures”. Clarendon Press. Oxford.

\section{publicaciones del IETcc/CSIC}

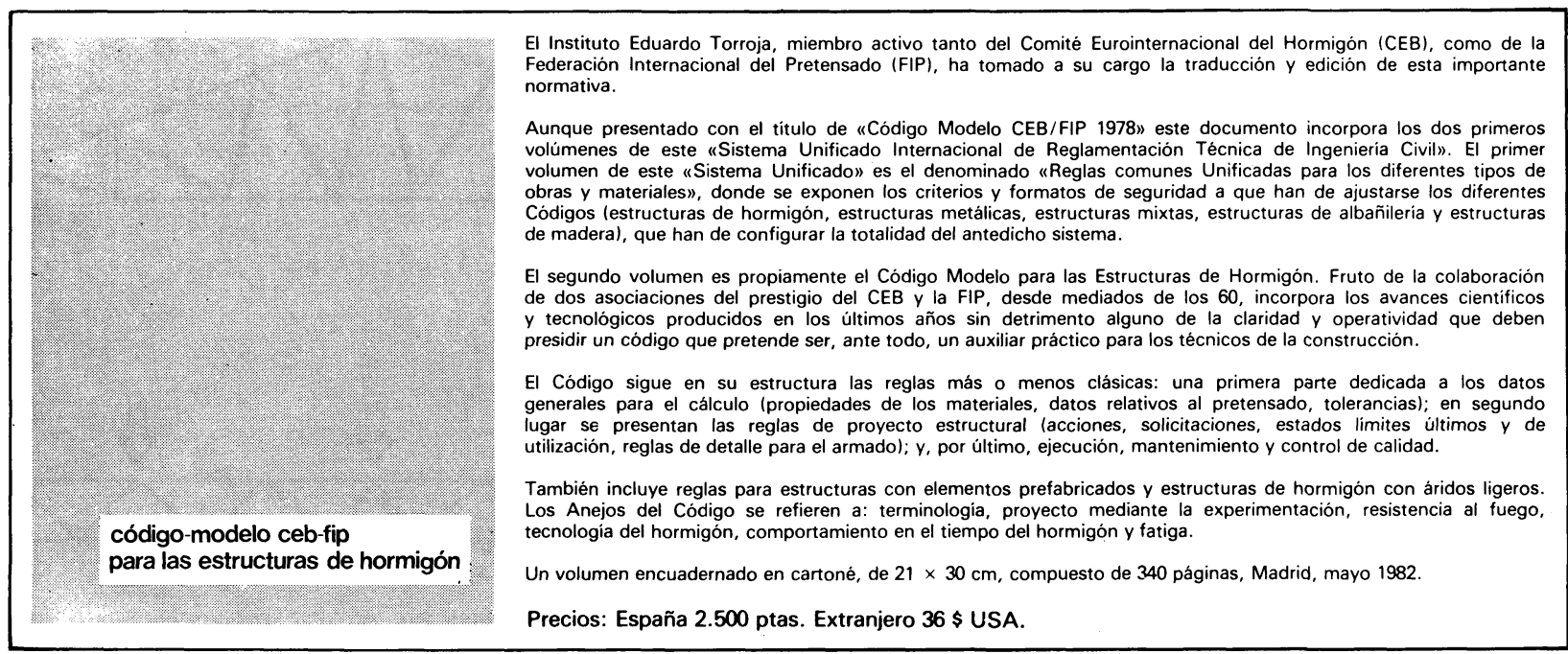

